\title{
Telehealth: Advances in Alternative Payment Models
}

Mei Zhao, PhD, Hanadi Hamadi, PhD, D. Rob Haley, PhD,

Jing $X u, P h D$, Cynthia White-Williams, PhD,

and Sinyoung Park, PhD

Department of Health Administration, Brooks College of Health, University of North Florida, Jacksonville, Florida, USA.

\begin{abstract}
Introduction: The hospital sector has shifted its focus to advanced information and communication technologies to facilitate health care delivery through telehealth services to alleviate the industry's most pressing challenges in quality care and access, especially under changing reimbursement payment approaches. The aim of this study was to examine the association between alternative payment models (APMs), market competition, and telehealth provisions in the hospital setting.
\end{abstract}

Materials and Methods: A secondary cross-sectional design to analyze 2018 census data of nonfederal short-term acute care hospitals in the United States was used. Multilevel logistic regressions models were used to analyze data from 4,257 hospitals across 1,874 counties. Counties with less than one hospital were excluded.

Results: Regarding APMs, we found that hospital participation in accountable care organizations and participation in a bundled payment risk arrangement are significantly associated with the provision of telehealth services. From the market perspective, competitive advantage was found to be statistically associated with hospitals providing telehealth services. In addition, other hospital characteristics such as ownership, part of a system, part of a network, and major teaching affiliation also have impact on the provision of telehealth.

Conclusions: The increase uptake of telehealth-related capabilities and their strong integration into care-delivery systems under APMs present exciting opportunities to enhance the merit of clinical care, and challenges as clinical professionals are not adept to using such technologies. There is a need to provide comprehensive of evidence on telehealth.

Keywords: telehealth, telemedicine, alternative payment model, accountable care organization, bundled payment

\section{Introduction}

elehealth is a way to provide health care services regardless of place, time, or physical barriers. ${ }^{1}$ It has emerged as an important component of the health care system, especially within the hospital setting, because it has shown to significantly impact hospitals in term of access, quality, and cost. ${ }^{2,3}$ Particularly, the use of telehealth technologies, tools, and services may increase patients' access to care, reduce unnecessary health care utilization and moral hazard, and increase hospitals' competitive advantage. ${ }^{1,4,5}$

Telehealth uses advanced information and communication technologies (ICT) to support clinical care delivery, patient-centered education, public health efforts, and administration. ${ }^{6-8}$ According to the Department of Health and Human Services (DHHS), $>40 \%$ of hospitals used telehealth interventions for their employees and patients, and telecommunicated with other health care professionals and among health care professionals and patients. ${ }^{7}$ There is an increased sense of urgency to advance evidence-based research for telehealth technology and use it as quickly to expand into multiple health sectors. ${ }^{9,10}$

One sector of health care that has increased its use of telehealth services is the hospital. Hospitals have shifted their focus to advanced ICT to facilitate health care delivery through telehealth services to alleviate the industry's most pressing challenges in quality care and access. ${ }^{11,12}$ Studies have shown that technology can improve patient outcomes, it may also increase hospital efficiency and financial performance especially under changing reimbursement payment approaches. ${ }^{13}$

\section{COMPETITIVE ADVANTAGE}

Hospitals may gain significant competitive advantage through ICT applications, yet the U.S. hospital industry lags behind other health care industries in terms of technology adoption and adaptation. ${ }^{14}$ According to the DHHS, only $40 \%$ of hospitals adopted telehealth interventions compared with $60 \%$ for other health care organizations, such as private physician practices and home health care. ${ }^{1,15}$ For urgentcare centers, telehealth utilization increased by $1,434 \%$ from 2008 to $2017 .{ }^{16}$ Reports indicated that telehealth adoption among hospitals and health systems has increased over the past 5 years from 54\% in 2014 to $85 \%$ in $2019 .{ }^{16}$ Two-way 
video/webcam technologies are being used by almost twothirds of hospitals in 2019, which has significantly increased from less than half in $2016 .{ }^{16,17}$

\section{ALTERNATIVE PAYMENT MODELS}

Hospital alternative payment models (APMs) have developed rapidly over the past several years. ${ }^{18}$ Two main alternative payment methods are bundled payments and accountable care organizations (ACOs). Bundling is a payment scheme that allows health care providers to receive a single payment for services rendered across one or parts of the care continuum. The ACO is a health care organization that links reimbursement to outcomes such as quality care and cost reductions reimbursements to quality metrics and reductions in the cost of care. ${ }^{19}$ The ACO is responsible to patients and third-party payers for the appropriateness and quality of care. The goal of the ACO was to promote efficiencies at all levels of patient care. Successes of the ACO are reflected in the cost saving among the provider participants in the program.

Organizations that have integrated their financial structure with their delivery of care using such APMs, cover and often encourage the utilization of ICT to improve care, reduce cost, and facilitate better and timely access to care. ${ }^{19}$ CMS grants more flexibility for telemedicine services under bundled payment model for joint replacements. ${ }^{20}$ However, more information is needed about the association between ACOs, bundled payment models, and telehealth adoption. Preliminary evidence suggests that hospitals that participate in an ACO may be more likely to adopt telehealth strategies to accommodate the increase of patient volume. ${ }^{19,21}$

\section{PURPOSE OF THIS STUDY}

Thus, the utilization of ICT in such an environment may be critical to ensure adequate access to care. The increased political push and use of bundled payments and ACO provide an opportunity to better understand how organizations are experimenting with telehealth to improve health. In addition, market characteristics also play a significant role for telehealth adoption. Hospitals located in more competitive areas and received reimbursement for private payers were associated with higher level of hospital telehealth adoption. ${ }^{4,22}$ Therefore, the aim of this study was to examine the association between APMs, market competition, and telehealth provisions in the hospital setting.

\section{Materials and Methods}

We used a secondary cross-sectional design to analyze census data of nonfederal short-term acute care hospitals in the United States.

\section{DATA SOURCES}

We used the 2018 American Hospital Association (AHA) Annual Survey, Area Health Resource File (AHRF), and Dartmouth Atlas Hospital Service Area (HSA) dataset. The HSA dataset provides geocodes for geographic boundaries of hospital service area $(n=3,234)$. HSA is defined as the collection of ZIP codes where residents receive the largest proportion of their hospitalization from the hospitals in that area. The AHA data collect information on $>6,000$ hospitals. The survey contains information specific to hospital characteristics including hospital structure and process. The AHRF collects and stores county-level information across the United States about health care professional, health facilities, hospital utilization, and population estimates. The three datasets were all merged using CMS Provider Number and County Federal Information Processing Standards (FIPS).

The data on hospital characteristics that support the findings of this study were obtained from the AHA. The Area Health Resource File and Dartmouth Atlas Hospital Referral Region are both publicly available datasets and can be downloaded at https://data.hrsa.gov/topics/health-workforce/ ahrf, and https://atlasdata.dartmouth.edu/static/research_data_ archive?tab=39, respectively.

\section{MEASURES}

Our dependent variable was hospital use of telehealth services $(1=$ Yes, $\mathrm{No}=0)$. We created an overall telehealth dichotomous variable using a series of six survey items in the 2018 AHA data. The survey asked hospitals whether they provided consultation and office visits, electronic intensive care unit, stroke care, psychiatric and addiction treatment, remote patient monitoring postdischarge, or remote patient monitoring ongoing chronic management through telehealth. If hospitals answered "yes" to providing any of the listed telehealth services, then they provided telehealth services $(n=2,351)$, and if hospitals answered "no" to all the listed services, then those hospitals did not provide any telehealth services $(n=1,906)$. The independent variables are hospital participating in ACOs $(1=\mathrm{Yes}, \mathrm{No}=0)$, participating in a bundled payment risk arrangement $(1=$ Yes, No $=0)$, and market competition, measured by the Herfindahl-Hirschman Index (HHI). A hospital HHI is the ratio of total hospital inpatient days by a county's total inpatient days accounting for a hospital's system affiliation. A hospital HHI closer to 1 represents a monopoly, whereas closer to 0 represents a competitive market.

In this study, we also included both hospital and market level control variables. Previous research shows that organization characteristics such as hospital size, system membership, 


\section{ZHAO ET AL.}

ownership, and teaching status are important to understanding the propensity to technology adoption. ${ }^{4,23}$ Hospital size (small: 0-99 beds, medium: 100-399 beds, large: 400+ beds) is an accepted factor in predicting organizational capacity. ${ }^{24}$ Thus, hospital characteristics that were included in the study based on past research contribution were hospital size (small: 0-99 beds, medium: 100-399 beds, large: 400+ beds), hospital ownership (for-profit, not-for-profit, and nonfederal government), teaching affiliation (major teaching, minor teaching, and nonteaching), hospital location (rural and urban), critical access hospital, part of a system, and part of a network. In addition, hospital payer mix is assessed by considering Medicaid discharges as a percentage of total discharges. Furthermore, we included several market covariates that were identified of importance in previous research. These variables included health professional shortage areas $(1=$ physician or mental health professional shortage county, $0=$ not a shortage county), per capita income, and population size. These variables will impact the market supply and demand. ${ }^{25}$

\section{ANALYSIS}

We summarized our findings using frequency and percentage for categorical variables and mean values and standard deviations for numeric variables. The analyses were performed using STATA 14 SE. Multilevel logistic regressions models were used to adjust for county nesting effect. This study analyzed data from 4,257 hospitals across 1,874 counties. That is, we have 4,257 hospitals (level 1 units) nested in 1,874 counties (level 2 units). Counties pertain to a level (rather than a predictor variable), whereas hospital characteristics such as size, ownership, location pertain to a predictor variable because its categories are both nonrandom and theoretically meaningful. Therefore, the multilevel logistic regression analysis considers the variations owing to nesting structure in the data and allows the examination of the effects of group-level (county) and individual-level variables (hospital) on individual-level outcomes. In addition, this analysis allows the examination of both between-group and within-group variability and how group-level and individual-level variables are related to variability at both levels. All variables were tested for multicollinearity. Akaike's and Schwarz's Bayesian information criteria were used to determine model fit.

\section{Results}

Our sample $(4,257)$ is representative of the U.S. hospital population, which includes almost all the nonfederal, shortterm general hospitals. Hospitals with missing data were not included in the study.

\section{DESCRIPTIVE STATISTICS}

Table 1 provides the percentage of hospitals that provided telehealth in 2018. Among all the community hospitals $(4,257)$ in the United States, 55.23\% used telehealth services $(n=2,351)$ in 2017 . The most common service provided is stroke care (39.56\%), followed by consulting and office visits (37.59\%) and psychiatric and addiction treatment (24.43\%). Other telehealth services are relatively less prevalent, with remote patient monitoring ongoing chronic care management (18.98\%), remote patient monitoring postdischarge (15.03\%), and electronic intensive care unit (17.27\%).

Table 2 provides the bivariate analysis of the independent variables to the dependent variable. The results show that the likelihood of using telehealth services is significantly correlated with the hospital participating in ACOs and participating in a bundled payment risk arrangement. In addition, the analysis indicates that the telehealth provision was significantly related to hospital market competition (HHI). Finally, hospital characteristics such as hospital size, ownership, part of a system, part of a network, teaching affiliation, location, whether is a critical access hospital and market characteristics such as the designated shortage county, and per capita income are all significantly related to the telehealth provision.

\begin{tabular}{|c|c|c|c|c|}
\hline \multirow[b]{3}{*}{ Telehealth services } & \multicolumn{4}{|c|}{ HOSPITAL PROVISION OF TELEHEALTH SERVICES $(N=4,257)$} \\
\hline & \multicolumn{2}{|c|}{ NO $(\%)$} & \multicolumn{2}{|c|}{ YES $(\%)$} \\
\hline & 1,906 & 44.77 & 2,351 & 55.23 \\
\hline Consultation and office visits & 2,657 & 62.41 & 1,600 & 37.59 \\
\hline Electronic intensive care unit & 3,522 & 82.73 & 735 & 17.27 \\
\hline Stroke care & 2,573 & 60.44 & 1,684 & 39.56 \\
\hline Psychiatric and addiction treatment & 3,217 & 75.57 & 1,040 & 24.43 \\
\hline Remote patient monitoring postdischarge & 3,617 & 84.97 & 640 & 15.03 \\
\hline $\begin{array}{l}\text { Remote patient monitoring ongoing } \\
\text { chronic care management }\end{array}$ & 3,449 & 81.02 & 808 & 18.98 \\
\hline
\end{tabular}




\section{Table 2. Descriptive Statistics of Telehealth Services Based on Hospital Characteristics}

HOSPITAL PROVISION OF TELEHEALTH SERVICES

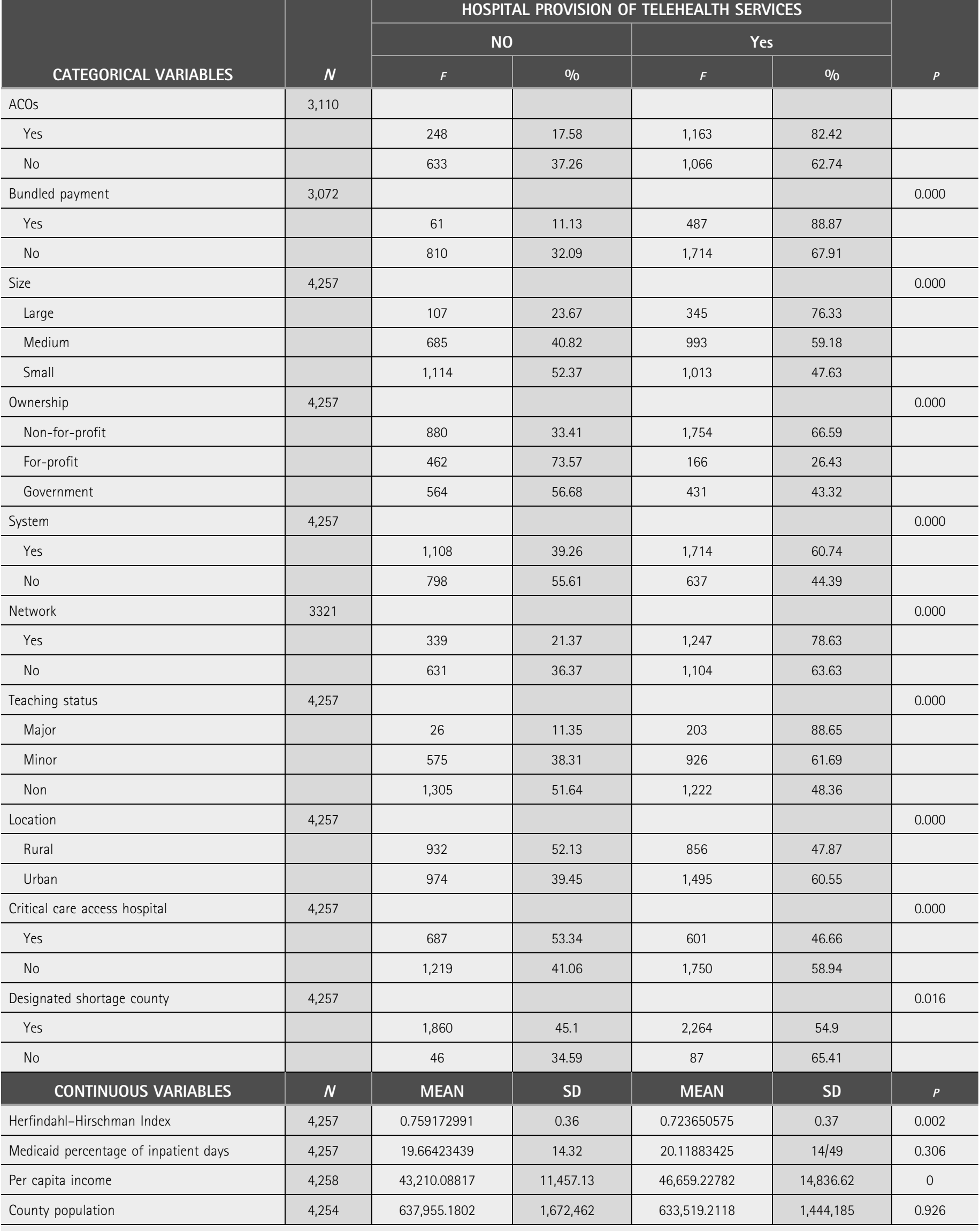

ACO, accountable care organization; SD, standard deviation. 


\section{ZHAO ET AL.}

\section{MULTIVARIATE ANALYSIS}

Table 3 provides the findings from the multilevel logistic regression model. As predicted, participation in ACOs and bundled payment risk arrangements are significantly positively related to hospital telehealth provision. In addition, the higher HHI, meaning the lower market competition, is significantly related to hospital telehealth provision. Regarding other hospital characteristics, the significant impacting factors are hospital ownership, part of a system, part of a network, and being a major teaching hospital. However, no other hospital and market characteristics are significant.

Compared with the private non-for-profit hospitals, both the government-owned and private for-profit hospitals are less likely to provide telehealth services. Compared with in-

Table 3. Multilevel Analysis of Telehealth Services, Hospital Characteristics, and Community Characteristics

\begin{tabular}{l|c|c|c|l}
\multicolumn{1}{l|}{} & ODDS RATIO & SD & $95 \%$ Cl & \multicolumn{1}{c}{$\boldsymbol{P > z}$} \\
\hline Part of an ACO (Reference: No) & 1.84 & 0.21 & $1.47-2.30$ & 0 \\
\hline Bundled payment (Reference: No) & 2.12 & 0.38 & $1.49-3.00$ & 0 \\
\hline Herfindahl-Hirschman Index & 1.57 & 0.27 & $1.12-2.21$ & 0.009 \\
\hline Hospital size (Reference: Small) & 1.33 & 0.19 & $1.00-1.77$ & 0.052 \\
\hline Medium & 1.50 & 0.36 & $0.94-2.40$ & 0.093 \\
\hline Large &
\end{tabular}

Ownership status (Reference: Not-for-Profit)

\begin{tabular}{l|l|l|l|l}
\hline Government & 0.51 & 0.06 & $0.40-0.66$ & 0 \\
\hline For Profit & 0.34 & 0.06 & $0.24-0.48$ & 0 \\
\hline Part of a system (Reference: No) & 1.30 & 0.15 & $1.03-1.64$ & 0.027 \\
\hline Part of a Network (Reference: No) & 1.41 & 0.15 & $1.15-1.75$ & 0.001 \\
\hline
\end{tabular}

\begin{tabular}{l|l|l|l|l}
\hline \multicolumn{5}{l|}{ Teaching affiliation (Reference: Nonteaching) } \\
\hline \multicolumn{1}{c|}{ Minor } & 0.95 & 0.12 & $0.74-1.23$ & 0.72 \\
\hline Major & 2.67 & 0.89 & $1.40-5.12$ & 0.003 \\
\hline Rural location (Reference: Urban) & 0.88 & 0.12 & $0.67-1.16$ & 0.361 \\
\hline Critical Access Hospital (Reference: No) & 0.79 & 0.11 & $0.60-1.03$ & 0.082 \\
\hline Designated shortage county (Reference: No) & 0.81 & 0.27 & $0.41-1.58$ & 0.537 \\
\hline Medicaid percentage of inpatient days & 1.00 & 0.003 & $0.99-1.01$ & 0.954 \\
\hline Per capita income & 1.00 & $5.16 \mathrm{E}-06$ & $1.00-1.00$ & 0.079 \\
\hline Population size & 0.99 & $7.86 \mathrm{E}-08$ & $1.00-1.00$ & 0.167 \\
\hline AIC & \multicolumn{5}{|l}{$3,269.83$} \\
\hline BIC & $3,384.3$ \\
\hline
\end{tabular}

AIC, Akanke information criteria; BIC, Bayesian information criteria; $\mathrm{Cl}$, confidence interval. dependent hospitals, the system-owned hospitals are more likely to provide telehealth services. Compared with the hospitals that are not in a network, the ones that are in a network are more likely to provide telehealth services. Compared with nonteaching hospitals, major teaching hospitals are more likely to provide telehealth services, whereas minor teaching hospitals are not significant.

The odds ratio estimates with 95\% Wald's confidence limits are provided in Table 3 to report the magnitudes of the impact from the predictors. The odds for hospitals that participate in an ACO is 1.84 times more than that of hospitals without participation to offer telehealth services. Similarly, the odds for hospitals that participate in bundled payment program is two times more than that of hospitals without participation. In addition, a unit increase from the average $\mathrm{HHI}$ leads to a 57\% increase in the odds to provide telehealth services. Finally, this study also shows that the odds for major teaching hospitals to provide telehealth services is 2.67 times more than that of nonteaching hospitals. On the contrary, participation in the system and network increases the odds by $\sim 30 \%$ and $41 \%$, respectively, whereas government and for-profit hospitals decreases the odds by $49 \%$ and $66 \%$ compared with not-for-profit hospitals.

\section{Discussion}

This study provides important insight into the association between APMs, market competition, and telehealth provision in the hospital setting. The economic efficiencies of telehealth are supported by recent studies but have not been studied with financial risk models such as ACOs and bundled payments. ${ }^{26,27}$ Our research found that hospitals participating in ACOs and those participating in bundled payment programs were more likely to provide telehealth services. These financing models encourage organizations to use specialist resources for patients in an efficient manner. ${ }^{28}$ This likely indicates that the economic efficiencies associated with telehealth interventions may be facilitating hospitals participating in higher risk financial models to adopt telehealth. Similar to our findings, a study of 393 ACO hospitals and 810 non-ACO hospitals found that ACO participating hospitals were more likely to adopt health IT. ${ }^{29}$ 
However, some authors suggest that ACO contracts that are driven by cost reductions may not readily adopt new technologies. Nevertheless, the need for cost reductions may incentivize ACOs to eventually adopt telehealth technology as the benefits are noted over time (years) compared with the cost of initial investments. ${ }^{30}$

Our study found that hospital market concentration has a significant effect on hospital telehealth provision. The findings indicate that hospitals located in less competitive markets were more likely to provide telehealth services. Although our research is in contrast to a previous study that found hospital competition was not associated with telehealth adoption, it did support other studies that hospitals located in less competitive areas were more likely to adopt telehealth.,31,32 Hospitals in less competitive markets are more likely to lack the specialist resources than that of more highly populated and competitive areas. Therefore, less populated and more rural areas would need to rely on telehealth to cost-effectively bring needed services into hard to staff areas. ${ }^{31}$ Furthermore, hospitals located in more competitive areas and those that received a greater percentage of reimbursement from private payers were associated with a higher level of hospital telehealth adoption. ${ }^{4,22}$

Other hospital characteristics were found to significantly influence the provision of telehealth. Our research indicates that, compared with private not-for-profit hospitals, both the government and for-profit hospitals were less likely to provide telehealth services. In addition, system-owned hospitals and hospitals in a network were more likely to provide telehealth services. This is supported by Ward et al. who studied the U.S. hospitals in the 2013 HIMSS database. Their study results indicated that hospitals that were more likely to have implemented telehealth services were not-for-profit institutions, academic medical centers, hospitals that were part of integrated delivery systems. ${ }^{23,29}$ Furthermore, another study revealed that large system-affiliated, not-for-profit, and teaching hospitals have a greater propensity to adopt telehealth programs. ${ }^{23}$ Not-for-profit hospitals were able to use excess income to fund patient benefits, and hospitals that were system affiliated had greater access to shared information and coordination of resources, as well as the ability to share risk and costs. ${ }^{23}$

Similarly, Adler-Milstein et al. researched 2,891 acute care U.S. hospitals using the IT Supplement to the AHA 2012 Annual Survey of Hospitals and found that hospitals that had greater technological capabilities were more likely to have adopted some type of telehealth. ${ }^{4}$ These were typically hospitals that were part of larger hospital systems and teaching hospitals. ${ }^{4}$ They also found that hospitals in large rural areas had a higher likelihood to be associated with telehealth adoption. In support, according to Huilgol et al. ${ }^{32}$ hospitals located in rural, less populated areas, with lower number of employees, and utilizing technology integrating into electronic health record adopted telehealth more than counterparts in California. Other studies found that hospitals located in remote and isolated regions were less likely to employ telehealth service. ${ }^{3,33}$

\section{POLICY AND PRACTICE IMPLICATIONS}

This study provides important insights into the association between APMs, market competition, and telehealth provisions in the hospital setting. By examining both hospital and market characteristics that determine hospital telehealth adoption using the latest national level data, this study provided a framework to incentivize payment models and promulgate policies to promote quality care using the latest technological advancements. Therefore, the findings from this study provide a more updated and systematic consideration for hospital telehealth adoption. Hospital administrators and policymakers need to better understand the financial efficiencies of telehealth services and the provision of health care. The recent changes in legislation will revise reimbursement and, therefore, impact hospitals adoption of telehealth services. Both the Health Information Technology for Economic and Clinical Health (HITECH) Act of 2009 and the Patient Accountability and Affordable Care Act (ACA) of 2010 supported the expansion of and innovation in telehealth technologies. The ACA supported telehealth in the context of ACOs that focused on attempting to foster evidence-based high-quality and coordinated care and cost saving (42 U.S.C. $\times 1395$ jjj). The Medicare Access and CHIP Reauthorization Act (MACRA) of 2015 has accelerated the shift from traditional fee-for-service payment model to merit and value-based payment scheme (Public Law No. 114-10), which has created the need to improve the approach used to deliver care. As our results support that value-based care and the expansion of APMs such as bundled payment and ACOs can facilitate the adoption of telehealth services. ${ }^{34}$ Telehealth services seems to be a cost-effective method to provide some aspects of care. Therefore, policy makers should understand how the various payment methodologies can impact the growth and dissemination of health care telehealth technologies and innovations.

Our research also found that for-profit hospitals were less likely to provide telehealth services than not-for-profit hospitals. Policy makers should understand why and develop policies that encourage all hospitals and health systems to use more cost-efficient, yet effective, methods of providing 
health care services. For example, policies that encourage hospital administrators to develop key performance indicators for telehealth services should be considered to lower the cost of care. Given that the U.S. health care system will continue to evolve toward improved quality and efficiency, hospitals and health systems must have the analytic capabilities to track and report how each of their telehealth services is performing. Key performance indicators for telehealth services should be developed and measured. This can improve efforts to better reimburse for telehealth services, as several legislative proposals have attempted to expand payment but are typically viewed by economists and budget experts as cost increasing if not implemented and utilized well.

\section{Acknowledgment}

This research did not receive any specific grant from funding agencies in the public, commercial, or not-for-profit sectors.

\section{Disclosure Statement}

No competing financial interests exist.

\section{Funding Information}

The University of North Florida's Department of Health Administration Marcus E. Drewa Distinguished Endowed Professorship provided funding for the study. The source was not involved in the study design, collection, analysis, or interpretation of data, or in preparation or submission of the manuscript for publication.

\section{REFERENCES}

1. Tuckson RV, Edmunds M, Hodgkins ML. Telehealth. N Engl J Med 2017;377: 1585-1592.

2. Bashshur RL, Reardon TG, Shannon GW. Telemedicine: A new health care delivery system. Annu Rev Public Health 2000;21:613-637.

3. Gagnon MP, Lamothe L, Fortin JP, Cloutier A, Godin G, Gagne C, et al. Telehealth adoption in hospitals: An organisational perspective. J Health Organ Manag 2005;19:32-56.

4. Adler-Milstein J, Kvedar J, Bates DW. Telehealth among us hospitals: Several factors, including state reimbursement and licensure policies, influence adoption. Health Aff (Millwood) 2014;33:207-215.

5. O'Connor M, Asdornwised U, Dempsey ML, Huffenberger A, Jost S, Flynn D, et al. Using telehealth to reduce all-cause 30-day hospital readmissions among heart failure patients receiving skilled home health services. App/ Clin Inform 2016;7:238-247.

6. Nandra K, Koenig G, DelMastro A, Mishler EA, Hollander JE, Yeo CJ. Telehealth provides a comprehensive approach to the surgical patient. Am J Surg 2019; 218:476-479.

7. Park J, Erikson C, Han X, lyer P. Are state telehealth policies associated with the use of telehealth services among underserved populations? Health Aff (Millwood) 2018;37:2060-2068.
8. The Office of the National Coordinator for Health Information Technology (ONC). Telemedicine and telehealth Washington, DC: HealthIT.gov; 2019 [cited September 28, 2019]. Available at https://www.healthit.gov/topic/health-itinitiatives/telemedicine-and-telehealth (last accessed November 1, 2019).

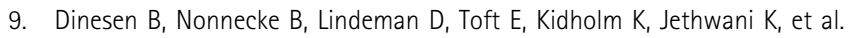
Personalized telehealth in the future: A global research agenda. J Med Internet Res 2016;18:e53.

10. Totten AM, Womack DM, Eden KB, McDonagh MS, Griffin JC, Grusing S, et al. Telehealth: Mapping the evidence for patient outcomes from systematic reviews. Rockville, MD: Agency for Healthcare Research and Quality, AHRO Comparative Effectiveness Technical Briefs. 2016; Report No.: 16-EHCO34-EF.

11. Abdalla YS. Critical factors determining adoption of telemedicine. Int J Online Engineer 2019;15.DOI: https://doi.org/10.3991/ijoe.v15i11.10492.

12. Behkami N, Daim TU. Exploring technology adoption in the case of the patientcentered medical home. Health Pol Technol 2016;5:166-188.

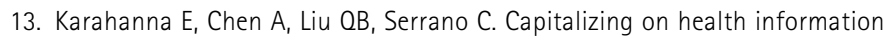
technology to enable digital advantage in U.S. hospitals. MIS Quarterly 2019;43:113-140.

14. Ahmadi $H$, Nilashi $M$, Shahmoradi L, Ibrahim 0 , Sadoughi $F$, Alizadeh $M$, et al. The moderating effect of hospital size on inter and intra-organizational factors of hospital information system adoption. Technol Forecast Soc Change 2018; 134:124-149.

15. Edmunds $M$, Tuckson $R$, Lewis J, Atchinson $B$, Rheuban $K$, Fanberg $H$, et al. An emergent research and policy framework for telehealth. EGEMS (Wash DC) 2017;5:1303.

16. Landi $\mathrm{H}$. Telehealth use jumps at inpatient facilities while outpatient adoption remains flat: Survey: Fierce Healthcare; 2019 [cited 2019 September 1]. Available at https://www.fiercehealthcare.com/tech/85-hospitals-usetelehealth-up-from-54-2014-survey (last accessed October 27, 2019).

17. Dayal P, Chang CH, Benko WS, Pollock BH, Crossen SS, Kissee J, et al. Hospital utilization among rural children served by pediatric neurology telemedicine clinics. JAMA Netw Open 2019;2:e199364.

18. Muhlestein D, Saunders R, Richards $R$, McClellan M. Recent progress in the value journey: Growth of acos and value-based payment models in 2018. Health Affairs Blog 2018;10.

19. Dorsey ER, Topol EJ. State of telehealth. N Engl J Med 2016;375:154-161.

20. Landi H. CMS grants more flexibility for telemedicine services under bundled payment model for joint replacements. Healthcare Innovation. 2015. Available at https://www.hcinnovationgroup.com/policy-value-based-care/news/13026008/ cms-grants-more-flexibility-for-telemedicine-services-under-bundled-paymentmodel-for-joint-replacements (last accessed October 26, 2019).

21. Pearl R. Kaiser permanente northern california: Current experiences with internet, mobile, and video technologies. Health Aff (Millwood) 2014;33:251-257.

22. Shea CM, Tabriz AA, Turner K, North S, Reiter KL. Telestroke adoption among community hospitals in north carolina: A cross-sectional study. J Stroke Cerebrovasc Dis 2018;27:2411-2417.

23. Asagbra $\mathrm{OE}$, Burke $\mathrm{D}$, Liang $\mathrm{H}$. Why hospitals adopt patient engagement functionalities at different speeds? A moderated trend analysis. Int J Med Inform 2018;111:123-130.

24. Yoon SN, Lee D, Schniederjans M. Effects of innovation leadership and supply chain innovation on supply chain efficiency: Focusing on hospital size. Technol Forecast Soc Change 2016;113:412-421.

25. Luo W. Using a gis-based floating catchment method to assess areas with shortage of physicians. Health Place 2004;10:1-11.

26. Licurse $A M$, Mehrotra $A$. The effect of telehealth on spending: Thinking through the numbers. Ann Intern Med 2018;168:737-738.

27. Yilmaz SK, Horn BP, Fore C, Bonham CA. An economic cost analysis of an expanding, multi-state behavioural telehealth intervention. J Telemed Telecare 2019:25:353-364. 
28. Kvedar J, Coye MJ, Everett W. Connected health: A review of technologies and strategies to improve patient care with telemedicine and telehealth. Health Aff (Millwood) 2014;33:194-199.

29. Walker DM, Mora AM, Scheck AM. Accountable care organization hospitals differ in health it capabilities. Am J Manag Care 2016;22: 802-807.

30. Trosman JR, Weldon CB, Douglas MP, Deverka PA, Watkins JB, Phillips KA. Decision making on medical innovations in a changing health care environment: Insights from accountable care organizations and payers on personalized medicine and other technologies. Value Health 2017;20: 40-46.

31. Ward MM, Merchant KA, Carter KD, Zhu X, Ullrich F, Wittrock $A$, et al. Use of telemedicine for ED physician coverage in critical access hospitals increased after cms policy clarification. Health Aff (Millwood) 2018;37: 2037-2044.

32. Huilgol YS, Miron-Shatz T, Joshi AU, Hollander JE. Hospital telehealth adoption increased in 2014 and 2015 and was influenced by population, hospital, and policy characteristics. Telemed J E Health 2019;26:455-461.

33. Evans RM. Telemedicine to treat traumatic brain injury in outlying and rural medical facilities: A review of current literature. Lynchburg J Med Sci 2019;1:16.
34. Osmonbekov T, Yordy $E_{1}$ Gregory $\mathrm{S}$. The affordable care act's impact on healthcare marketing. JL Bus Ethics 2014;20:21.

Address correspondence to: Hanadi Hamadi, PhD Department of Health Administration Brooks College of Health

University of North Florida Building 39, Room 4017 1 UNF Drive

Jacksonville, FL 32224-7699

USA

E-mail: h.hamadi@unf.edu

Received: November 28, 2019

Accepted: January 2, 2020

Online Publication Date: February 26, 2020 